\title{
Preoperative Cachexia predicts poor outcomes in young rather than elderly gastric cancer patients: a prospective study
}

This article was published in the following Dove Press journal: Cancer Management and Research

\author{
Xiaodong Chen ${ }^{1} *$ \\ Yunpeng Zeng ${ }^{1} * *$ \\ Yunshi Huang ${ }^{2}$ \\ Jingxuan Xu (1D \\ Weiyang Meng ${ }^{3}$ \\ Xiang Wang' \\ Ce Zhu ${ }^{2}$ \\ Guanbao Zhu ${ }^{2}$ \\ Chenchen Mao (iD) \\ Xian Shen ${ }^{1,2}$ \\ 'Department of Gastrointestinal Surgery, \\ The Second Affiliated Hospital, Wenzhou \\ Medical University, Wenzhou, Zhejiang, \\ People's Republic of China; ${ }^{2}$ Department \\ of Gastrointestinal Surgery, The First \\ Affiliated Hospital, Wenzhou Medical \\ University, Wenzhou, Zhejiang, People's \\ Republic of China; ${ }^{3}$ Department of \\ Emergency Medical, The Second \\ Affiliated Hospital, Wenzhou Medical \\ University, Wenzhou, Zhejiang, People's \\ Republic of China
}

*These authors contributed equally to this work

\begin{abstract}
Background: Cachexia affects nearly $50-80 \%$ of cancer patients, and most studies have only focused on elderly patients. We investigated preoperative cachexia in gastric cancer (GC) patients by age group and comprehensively analyzed the impact of preoperative cachexia on the prognosis of GC patients in all age groups.

Methods: In total, 575 patients were prospectively analyzed. The effect of preoperative cachexia on overall survival (OS) in all the patients and in patients with different age groups were investigated using log-rank test and Cox proportional hazards regression, respectively. Results: In total, 35.8\% (206 of 575) individuals were diagnosed with cachexia. The median survival of cachexia patients (29.2 months) was shorter than that of non-cachexia patients (35.7 months). Cachexia ( $\mathrm{HR}=1.976, P<0.001)$, age $(\mathrm{HR}=1.811, P<0.001)$, readmission (HR $=2.559, P<0.001)$, tumor size $(\mathrm{HR}=1.639, P=0.003)$, TNM stage (stage II: HR =2.215, $P=0.017$; stage III: $\mathrm{HR}=5.758, P<0.001)$, whole stomach cancer $(\mathrm{HR}=2.639, P<0.001)$, and combined operation ( $\mathrm{HR}=1.598, P=0.032$ ) were independently associated with worse OS. After grouping by age, cachexia was associated with OS in patients younger than 50 years old (HR $=4.947, P=0.029)$, patients $51-60$ years old $(\mathrm{HR}=2.232, P=0.026)$, and patients $61-70$ years old $(\mathrm{HR}=1.806, P=0.032)$, but not in patients older than 71 years $(\mathrm{HR}=1.411, P=0.119)$. Further, cachexia only significantly affected the postoperative length of stay $(P=0.015)$ and hospitalization costs $(P=0.032)$ in patients younger than 50 years old.
\end{abstract}

Conclusions: Preoperative cachexia predicts poor outcome in younger GC patients, and greater attention should be paid to these patients.

Keywords: cachexia, gastric cancer, overall survival, younger patients

\section{Introduction}

Cancer cachexia, also known as cancer-related wasting syndrome and cancer anorexia-cachexia syndrome, is a multifactorial syndrome defined by an ongoing loss of skeletal muscle mass, with or without loss of fat mass, that cannot be fully reversed by conventional nutritional support, leading to progressive functional impairment. ${ }^{1}$ It is also an indicator of tumor progression in patients with malignancy. ${ }^{2}$ Recently, cachexia has been receiving increasing attention because of its high prevalence, affecting nearly $50-80 \%$ of cancer patients, depending on the tumor type. ${ }^{3}$ Further, it may be the leading cause of nearly $20 \%$ of cancer deaths. ${ }^{3,4}$

The inability to prevent weight loss through nutritional intervention is one of several important distinctions between cancer cachexia and simple starvation. ${ }^{1}$ Moreover, the co-occurrence of cancer, especially gastrointestinal cancer, and
Correspondence: Chenchen Mao; Xian Shen

Department of Gastrointestinal Surgery, The Second Affiliated Hospital of Wenzhou Medical University, No. 109

West College Road, Wenzhou, Zhejiang Province, People's Republic of China

Tel +8605778 8002709

Email 578I17567@qq.com;

shenxian5166@gmail.com 
cachexia greatly weakens the patient's ability to recover. Bachmann et al demonstrated that pancreatic cancer patients with cachexia experienced a greater decline in survival, ${ }^{5}$ and Fukuta et al reported that preoperative cachexia greatly increased the postoperative length of stay in elderly patients with gastrointestinal cancer. ${ }^{6}$ Another report stated that the risk of inpatient death was higher for gastric cancer (GC) patients with cachexia. ${ }^{7}$ However, few studies have focused on the impact of cachexia on the long-term survival of GC patients, and most such studies only examined elderly patients.

Therefore, the relationship between GC and cachexia still needs to be fully elucidated to develop effective therapeutic strategies that consider this relationship. This study aimed to comprehensively evaluate the potential prognostic utility of cachexia in GC patients, including examining the differences between younger and elderly patients.

\section{Materials and methods}

\section{Patients}

Data were prospectively collected from 578 patients with GC who underwent subtotal gastrectomy at the Gastrointestinal Surgical Departments of The Second Affiliated Hospital of Wenzhou Medical University and The First Affiliated Hospital of Wenzhou Medical University in China between January 2014 and December 2016. Three patients who lacked imaging data were excluded. Current study was carried out in accordance with the Declaration of Helsinki, and the study protocol was approved by the ethics committees of the Second Affiliated Hospital of Wenzhou Medical University and the First Affiliated Hospital of Wenzhou Medical University.

\section{Diagnosis of cancer cachexia}

The definition of cachexia was $>5 \%$ loss of stable body weight over the previous 6 months, a body mass index (BMI) $<20 \mathrm{~kg} / \mathrm{m} 2$ and ongoing weight loss $>2 \%$, or sarcopenia and ongoing weight loss $>2 \%$. ${ }^{1}$ Specifically, low muscle mass was defined as a skeletal muscle mass index of $<7 \mathrm{~kg} / \mathrm{m} 2$ for men and $<5.7 \mathrm{~kg} / \mathrm{m} 2$ for women. ${ }^{6}$ Muscle mass was assessed using multifrequency bioelectrical impedance with eight tactile electrodes (InBody 430; Inbody Japan, Tokyo, Japan).

\section{Statistical analysis}

The Kolmogorov-Smirnov test was performed to assess the distribution equality of continuous parameters. Normally distributed data are presented as means \pm standard deviations (SDs), and non-normally distributed data are presented as medians and interquartile ranges (IQRs). In univariate analyses, the independent $t$-test and Mann-Whitney U-test were used to analyze intergroup differences in continuous variables, and the chi-square test and Fisher's exact test were applied to categorical variables. Overall survival (OS) was defined as the time between the date of diagnosis and the date of death or last known follow-up. The Kaplan-Meier method and log-rank test were used to estimate and compare survival based on specific factors. The Cox proportional hazard model was used to estimate the risk ratio in univariate and multivariate analyses. All $P$-values were two-sided, and $P<0.05$ was considered statistically significant. All statistical analyses were performed using SPSS software (version 22.0; SPSS Inc., Chicago, IL, USA).

\section{Results}

\section{Patient characteristics}

A total of 575 patients were enrolled in the present study. The baseline characteristics of these patients are shown in Table 1. Most patients were men $(n=433,75.3 \%)$, and the mean age of all the patients was 64.41 years $(\mathrm{SD}=10.6)$. The median BMI was $22.21 \mathrm{~kg} / \mathrm{m} 2$ (IQR =20.20-24.22). The mean PLR (platelet/lymphocyte ratio) and NLR (neutrophil/lymphocyte ratio) were $169.87(\mathrm{SD}=10.6)$ and $2.71(\mathrm{SD}=2.0)$, respectively. Of the 575 patients analyzed, $206(35.8 \%)$ were diagnosed with cancer cachexia.

\section{Correlation of clinicopathologic characteristics with cachexia}

The chi-square test, independent $t$-test, and Mann-Whitney U-test were used to examine the relationship between clinical characteristics and cachexia. Age $(P<0.001)$, BMI $(P<0.001)$, PLR $(P=0.001)$, NLR $(P<0.001)$, skeletal muscle mass $(P<0.001)$, grip strength $(P<0.001)$, preoperative hemoglobin $(P<0.001)$, preoperative albumin $(P<0.001)$, ASA $(P<0.001)$, Charlson comorbidity index (CCI) $(P<0.001)$, preoperative bleeding $(P=0.006)$, preoperative obstruction $(P<0.001)$, tumor size $(P=0.016)$, pathologic type $(P<0.001)$, and TNM stage $(P<0.001)$ were significantly correlated with cachexia. Further, the postoperative length of stay was longer, and the total hospitalization cost was higher in the cachexia group (Table 1). There was no significant association between cachexia and gender, visceral fat area, stride speed, histopathological differentiation, surgical bleeding, or abdominal surgery history. 
Table I Demographics, patient characteristics in overall study population and by cachexia group

\begin{tabular}{|c|c|c|c|c|}
\hline Factors & Total $(n=575)$ & Non-cachexia $(n=369)$ & Cachexia $(n=206)$ & $P$-value \\
\hline Gender & & & & 0.668 \\
\hline Male & $433(75.3 \%)$ & 280 (75.9\%) & $153(74.3 \%)$ & \\
\hline Female & $142(24.7 \%)$ & 89 (24.1\%) & $53(25.7 \%)$ & \\
\hline Age (y) & $64.41 \pm 10.6$ & $63.24 \pm 10.4$ & $66.49 \pm 10.8$ & $<0.00 I^{*}$ \\
\hline BMI (kg/m2) & $22.21(20.20-24.22)$ & $22.50(20.57-24.61)$ & $21.51(19.54-23.7)$ & $<0.00 I^{*}$ \\
\hline PLR & $169.87 \pm 95.8$ & $159.94 \pm 92.1$ & $187.70 \pm 99.9$ & $0.00 I^{*}$ \\
\hline NLR & $2.71 \pm 2.0$ & $2.56 \pm 1.6$ & $3.17 \pm 2.5$ & $<0.00 I^{*}$ \\
\hline Skeletal muscle $(\mathrm{cm} 2)$ & $41.98(35.86-47.93)$ & $42.92(37.45-48.90)$ & $39.52(34.43-46.99)$ & $<0.00 I^{*}$ \\
\hline Grip strength (kg) & $28.90(22.0-35.5)$ & $30.25(23.4-41.1)$ & $26.00(19.9-32.9)$ & $<0.00 I^{*}$ \\
\hline Stride speed $(\mathrm{m} / \mathrm{s})$ & $1.00 \pm 0.2$ & $0.99 \pm 0.2$ & $0.96 \pm 0.3$ & 0.246 \\
\hline Preoperative hemoglobin & $120.83 \pm 22.0$ & $124.15 \pm 2||$. & $1 \mid 4.87 \pm 22.5$ & $<0.00 I^{*}$ \\
\hline Preoperative albumin & $38.50(34.95-41.50)$ & $39.20(35.70-41.90)$ & $37.20(33.65-40.2)$ & $<0.00 I^{*}$ \\
\hline ASA & & & & $0.001 *$ \\
\hline $\mathrm{I}-2$ & 475 (82.6\%) & $319(86.4 \%)$ & $156(75.7 \%)$ & \\
\hline $3-4$ & $100(17.4 \%)$ & $50(13.6 \%)$ & $50(24.3 \%)$ & \\
\hline Charison scroe & & & & $<0.00 I^{*}$ \\
\hline 0 & $293(51.0 \%)$ & $214(58.0 \%)$ & 79 (38.3\%) & \\
\hline $1-3$ & $260(45.2 \%)$ & 149 (40.4\%) & III (53.9\%) & \\
\hline $4-6$ & $22(3.8 \%)$ & $6(1.6 \%)$ & $16(7.8 \%)$ & \\
\hline Preoperative anaemia & & & & $<0.00 I^{*}$ \\
\hline No & $475(82.6 \%)$ & $322(87.3 \%)$ & $153(74.3 \%)$ & \\
\hline Yes & $100(13.4 \%)$ & 47 (12.7\%) & $53(25.7 \%)$ & \\
\hline Preoperative bleeding & & & & $0.006 *$ \\
\hline No & 487 (84.7\%) & 324 (87.8\%) & $163(79.1 \%)$ & \\
\hline Yes & 88 (I5.3\%) & 45 (12.2\%) & 43 (20.9\%) & \\
\hline Preoperative obstruction & & & & $<0.00 I^{*}$ \\
\hline No & 521 (90.6\%) & $36 \mid(97.8 \%)$ & $160(77.7 \%)$ & \\
\hline Yes & $54(9.4 \%)$ & $8(0.2 \%)$ & $46(22.3 \%)$ & \\
\hline Tumor location & & & & $0.04 *$ \\
\hline Cardia & $73(12.7 \%)$ & 39 (10.6\%) & $34(16.5 \%)$ & \\
\hline Body & 116 (20.2\%) & 77 (20.9\%) & 39 (18.9\%) & \\
\hline Antrum & $364(63.3 \%)$ & $243(65.9 \%)$ & $121(58.7 \%)$ & \\
\hline Total & $22(3.8 \%)$ & $10(2.6 \%)$ & $12(5.9 \%)$ & \\
\hline Tumor size & & & & $0.016 *$ \\
\hline$\leq 4.75$ & $372(64.7 \%)$ & 252 (68.3\%) & $120(58.3 \%)$ & \\
\hline$>4.75$ & $203(35.3 \%)$ & $117(31.7 \%)$ & $86(41.7 \%)$ & \\
\hline Histopathological differentiation & & & & 0.091 \\
\hline Differentiated & $422(73.4 \%)$ & $273(74.0 \%)$ & 149 (72.3\%) & \\
\hline Undifferentiated & $51(8.9 \%)$ & $26(7.0 \%)$ & 25 (I2.2\%) & \\
\hline Signet ring cell carcinoma & $102(17.7 \%)$ & $70(19.0 \%)$ & $32(\mid 5.5 \%)$ & \\
\hline
\end{tabular}


Table I (Continued).

\begin{tabular}{|c|c|c|c|c|}
\hline Factors & Total $(n=575)$ & Non-cachexia $(n=369)$ & Cachexia $(n=206)$ & $P$-value \\
\hline Pathologic type & & & & $<0.00 I^{*}$ \\
\hline Ulcerative type & 504 (87.7\%) & 337 (91.3\%) & $167(81.1 \%)$ & \\
\hline Non-ulcerative type & $7 \mid(12.3 \%)$ & $32(8.7 \%)$ & 39 (I8.9\%) & \\
\hline TNM stage & & & & $<0.00 I^{*}$ \\
\hline 1 & $185(32.2 \%)$ & 145 (39.3\%) & 40 (19.4\%) & \\
\hline II & $124(21.6 \%)$ & $76(20.6 \%)$ & $48(23.3 \%)$ & \\
\hline III & $266(46.2 \%)$ & 148 (40.1\%) & II 8 (57.3\%) & \\
\hline Surgical bleeding & & & & 0.066 \\
\hline No & $500(87.0 \%)$ & 328 (88.9\%) & I 72 (83.5\%) & \\
\hline Yes & 75 (I3.0\%) & $41(11.1 \%)$ & $34(16.5 \%)$ & \\
\hline Operation time & & & & 0.673 \\
\hline$\leq 210 \mathrm{~min}$ & $311(54.1 \%)$ & $202(54.7 \%)$ & 109 (52.9\%) & \\
\hline$>210 \mathrm{~min}$ & 264 (45.9\%) & $167(45.3 \%)$ & 97 (47.1\%) & \\
\hline Abdominal surgery history & & & & 0.38 \\
\hline No & $506(88.0 \%)$ & $328(88.9 \%)$ & I 78 (86.4\%) & \\
\hline Yes & $69(12.0 \%)$ & $41(11.1 \%)$ & $28(13.6 \%)$ & \\
\hline Length of stay (d) & $15.77 \pm 9.36$ & $14.97 \pm 9.39$ & $17.19 \pm 9.17$ & 0.006 \\
\hline Total cost (yuán) & $64,297.95 \pm 30,643.86$ & $61,791.02 \pm 32,846.58$ & $68,788.52 \pm 25,709.93$ & 0.009 \\
\hline
\end{tabular}

Notes: Data are presented as $n(\%)$, mean \pm SD, or median $[I Q R]$ unless otherwise indicated. *Statistically significant $(P<0.05)$.

Abbreviations: BMI, body mass index; ASA, American Society of Anaesthesiologists; NLR, neutrophil/lymphocyte ratio; PLR, platelet/lymphocyte ratio; TNM, TumorLymph, Node, Metastasis.

\section{Cachexia was independently associated with worse OS in GC patients}

As shown in Figure 1, patients with cachexia had a poor outcome: the median survival time in patients with cachexia (29.2 months) was shorter than in those without (35.7 months) $(P<0.001)$. On univariate analysis, cachexia was also associated with worse OS (hazard ratio [HR] 1.976, 95\% confidence interval $[\mathrm{CI}] 1.471-2.653, P<0.001)$. Additionally, age (HR 2.107, 95\% CI 1.569-2.830, $P<0.001$ ), lower BMI (HR 1.781, 95\% CI 1.120-2.834, $P=0.015$ ), higher ASA stage (HR 1.654, 95\% CI 1.1732.332, $P=0.004$ ), higher CCI (CCI 1-3: HR 1.412, 95\% CI $1.041-1.914, P=0.026$; CCI 4-6: HR 2.133, 95\% CI $1.103-$ 4.126, $P=0.024$ ), lower preoperative grip strength (HR 1.858, 95\% CI 1.365-2.530, $P<0.001$ ), preoperative anemia (HR $1.520,95 \%$ CI $1.072-2.155, P=0.019)$, preoperative hypoalbuminemia (HR 2.344, 95\% CI 1.272-4.320, $P=0.006$ ), readmission (HR 1.940, 95\% CI 1.218-3.089, $P=0.005$ ), larger tumor size (HR 2.830, 95\% CI 2.102-3.810, $P<0.001$ ), higher TNM stage (stage II: HR $2.747,95 \% \mathrm{CI}$ 1.480-5.098, $P<0.001$; stage III: HR 7.823, 95\% CI 4.65413.149, $P<0.001$ ), anastomotic method (Billroth II: HR

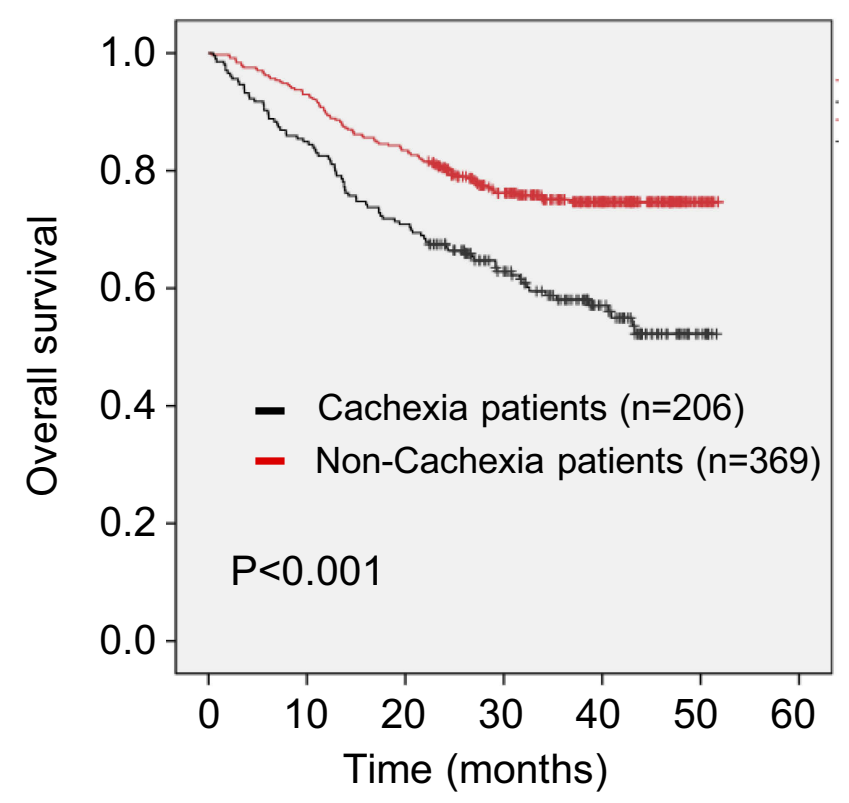

Figure I Kaplan-Meier curve for overall survival (OS) in patients with and without cachexia.

2.612, 95\% CI 1.661-4.108, $P<0.001$; Roux-en-Y: HR $2.909,95 \%$ CI $2.004-4.224, P<0.001)$, intraoperative blood transfusion (HR 2.844, 95\% CI 1.833-4.411, $P<0.001$ ), 
Table 2 COX regression analysis of the relationship between OS and cachexia or patient characteristics

\begin{tabular}{|c|c|c|c|c|}
\hline \multirow[t]{2}{*}{ Factors } & \multicolumn{2}{|l|}{ Univariate } & \multicolumn{2}{|l|}{ Multivariate } \\
\hline & HR (95\% Cl) & $P$-value & HR (95\% Cl) & $P$-value \\
\hline \multicolumn{5}{|l|}{ Gender } \\
\hline Male & Reference & & & \\
\hline Female & $1.292(0.899-1.856)$ & 0.166 & & \\
\hline \multicolumn{5}{|l|}{ Age } \\
\hline$<70$ & Reference & & Reference & \\
\hline$\geq 70$ & $2.107(1.569-2.830)$ & $<0.001 *$ & 1.811 (1.334-2.458) & $<0.00 I^{*}$ \\
\hline \multicolumn{5}{|l|}{ BMI (kg/m2) } \\
\hline $18.5-23.5$ & Reference & & & \\
\hline$<18.5$ & 1.781 (1.120-2.834) & $0.015^{*}$ & & \\
\hline$>23.5$ & $0.949(0.686-1.313)$ & 0.752 & & \\
\hline \multicolumn{5}{|l|}{ ASA } \\
\hline $\mathrm{I}-2$ & Reference & & & \\
\hline $3-4$ & $1.654(1.173-2.332)$ & $0.004 *$ & & \\
\hline \multicolumn{5}{|l|}{ Charison scroe } \\
\hline 0 & Reference & & & \\
\hline $1-3$ & $1.412(1.04 \mid-1.914)$ & $0.026 *$ & & \\
\hline 4-6 & $2.133(1.103-4.126)$ & $0.024 *$ & & \\
\hline \multicolumn{5}{|l|}{ PLR } \\
\hline$\leq|3| . \mid$ & Reference & & & \\
\hline$>131.1$ & $1.336(0.978-1.825)$ & 0.068 & & \\
\hline \multicolumn{5}{|l|}{ NLR } \\
\hline$\leq 2.28$ & Reference & & & \\
\hline$>2.28$ & $1.482(1.101-1.994)$ & $0.009^{*}$ & & \\
\hline \multicolumn{5}{|c|}{ Preoperative Grip strength } \\
\hline Normal & Reference & & & \\
\hline Low & $1.858(1.365-2.530)$ & $<0.001 *$ & & \\
\hline \multicolumn{5}{|c|}{ Preoperative anemia } \\
\hline No & Reference & & & \\
\hline Yes & $1.520(1.072-2.155)$ & $0.019 *$ & & \\
\hline \multicolumn{5}{|c|}{ Preoperative hypoalbuminemia } \\
\hline No & Reference & & & \\
\hline Yes & $2.344(1.272-4.320)$ & $0.006 *$ & & \\
\hline \multicolumn{5}{|l|}{ Surgery history } \\
\hline No & Reference & & & \\
\hline Yes & $1.275(0.835-1.946)$ & 0.260 & & \\
\hline \multicolumn{5}{|l|}{ Readmission } \\
\hline No & Reference & & Reference & \\
\hline Yes & $1.940(1.218-3.089)$ & $0.005^{*}$ & $2.559(1.562-4.191)$ & $<0.00 I^{*}$ \\
\hline \multicolumn{5}{|l|}{ Cachexia } \\
\hline No & Reference & & Reference & \\
\hline Yes & $1.976(1.47 I-2.653)$ & $<0.00 I^{*}$ & $1.456(1.070-1.98 I)$ & $0.017^{*}$ \\
\hline
\end{tabular}

(Continued) 
Table 2 (Continued).

\begin{tabular}{|c|c|c|c|c|}
\hline \multirow[t]{2}{*}{ Factors } & \multicolumn{2}{|l|}{ Univariate } & \multicolumn{2}{|l|}{ Multivariate } \\
\hline & HR (95\% CI) & $P$-value & HR (95\% CI) & $P$-value \\
\hline \multicolumn{5}{|l|}{ Tumor size } \\
\hline$\leq 4.75$ & Reference & & Reference & \\
\hline$>4.75$ & $2.830(2.102-3.810)$ & $<0.00 I^{*}$ & $1.639(1.187-2.261)$ & $0.003^{*}$ \\
\hline \multicolumn{5}{|c|}{ Histopathological differentiation } \\
\hline Differentiated & Reference & & & \\
\hline Undifferentiated & $1.519(0.948-2.434)$ & 0.082 & & \\
\hline \multicolumn{5}{|l|}{ Pathologic type } \\
\hline Ulcerative type & Reference & & & \\
\hline Non-ulcerative type & $0.712(0.473-1.073)$ & 0.105 & & \\
\hline \multicolumn{5}{|l|}{ TNM stage } \\
\hline 1 & Reference & & Reference & \\
\hline ॥ & $2.747(1.480-5.098)$ & $<0.00 I^{*}$ & $2.215(1.155-4.248)$ & $0.017 *$ \\
\hline III & $7.823(4.654-13.149)$ & $<0.00 I^{*}$ & $5.758(3.285-10.094)$ & $<0.00 I^{*}$ \\
\hline \multicolumn{5}{|l|}{ Tumor location } \\
\hline Cardia & Reference & & Reference & \\
\hline Body & $0.940(0.548-1.611)$ & 0.821 & $1.044(0.6-1.817)$ & 0.878 \\
\hline Antrum & $0.94 \mid(0.594-1.489)$ & 0.795 & $1.118(0.697-1.793)$ & 0.643 \\
\hline Total & $4.125(2.159-7.879)$ & $<0.00 I^{*}$ & $2.639(1.358-5.130)$ & $<0.00 I^{*}$ \\
\hline \multicolumn{5}{|l|}{ Anastomotic method } \\
\hline Billroth I & Reference & & & \\
\hline Billroth II & $2.612(1.661-4.108)$ & $<0.00 I^{*}$ & & \\
\hline Roux-en-y & $2.909(2.004-4.224)$ & $<0.00 I^{*}$ & & \\
\hline \multicolumn{5}{|c|}{ Intraoperative blood transfusion } \\
\hline No & Reference & & & \\
\hline Yes & $2.844(1.833-4.4 I I)$ & $<0.00 I^{*}$ & & \\
\hline \multicolumn{5}{|l|}{ Intraoperative bleeding } \\
\hline No & Reference & & & \\
\hline Yes & $2.835(1.813-4.434)$ & $<0.00 I^{*}$ & & \\
\hline \multicolumn{5}{|c|}{ Postoperative complications above grade 2} \\
\hline No & Reference & & & \\
\hline Yes & $2.095(1.526-2.877)$ & $<0.00 I^{*}$ & & \\
\hline \multicolumn{5}{|l|}{ Combined operation } \\
\hline No & Reference & & Reference & \\
\hline Yes & $2.284(1.515-3.444)$ & $<0.00 I^{*}$ & $1.598(1.042-2.449)$ & $0.032^{*}$ \\
\hline
\end{tabular}

Notes: Data are presented as median [IQR]. *Statistically significant $(P<0.05)$.

Abbreviations: BMI, body mass index; ASA, American Society of Anaesthesiologists; NLR, neutrophil/lymphocyte ratio; PLR, platelet/lymphocyte ratio; TNM, TumorLymph, Node, Metastasis.

intraoperative bleeding (HR 2.835, 95\% CI 1.813-4.434, $P<0.001$ ), postoperative complications above grade 2 (HR $2.095,95 \%$ CI $1.526-2.877, P<0.001)$, and combined operation (HR 2.284, 95\% CI 1.515-3.444, $P<0.001$ ) were associated with poor OS. Furthermore, whole stomach cancer (HR 4.125, 95\% CI 2.159-7.879, $P<0.001$ ) and a higher
NLR (HR 1.482, 95\% CI 1.101-1.994, $P=0.009$ ) were also associated with worse OS (Table 2). No other clinicopathologic factors were statistically linked to outcome. On multivariate analysis, cachexia (HR 1.456, 95\% CI 1.070-1.981, $P=0.017)$, age (HR 1.811, 95\% CI 1.334-2.458, $P<0.001$ ), readmission (HR 2.559, 95\% CI 1.562-4.191, $P<0.001$ ), 
tumor size (HR 1.639, 95\% CI 1.187-2.261, $P=0.003$ ), TNM stage (stage II: HR 2.215, 95\% CI 1.155-4.248, $P=0.017$; stage III: HR 5.758, 95\% CI 3.285-10.094, $P<0.001$ ), whole stomach cancer (HR 2.639, 95\% CI 1.358-5.130, $P<0.001$ ), and combined operation (HR 1.598, 95\% CI 1.042-2.449, $P=0.032)$ were independently associated with worse OS (Table 2).

\section{Cachexia was associated with worse prognosis in younger patients}

As shown in Figure 2, of the 575 GC patients, most patients were $50-80$ years old. There were $29.4 \%(5 / 17)$, $27.8 \%$ (10/36), 34.1\% (46/135), 28.3\% (58/205), 45.7\% $(74 / 162)$, and $65.0 \%(13 / 20)$ of patients younger than 40 , $41-50,51-60,61-70,71-80$ and older than 81 age groups, respectively, diagnosed with cachexia.

As only 17 patients were younger than 40 years old and 20 patients were older than 80 years old, we further subdivided the patients into 4 groups, namely, patients aged younger than 50 (group I), patients 51-60 years old (group II), patients 61-70 years old (group III), and patients older than 71 (group IV). Interestingly, cachexia was able to predict poor outcome in the younger patients. The median survival in patients with cachexia was statistically shorter than that in those without cachexia in group I $(P=0.015)$, group II $(P=0.022)$, and group III $(P=0.029)$. Although the median survival was also shorter in cachexia patients in group IV, no statistical differences were found $(P=0.117)$ (Figure 3A). Additionally, as shown in Table 3, the effect of cachexia on prognosis decreased as age increased, and cachexia was a more effective risk factor for survival in

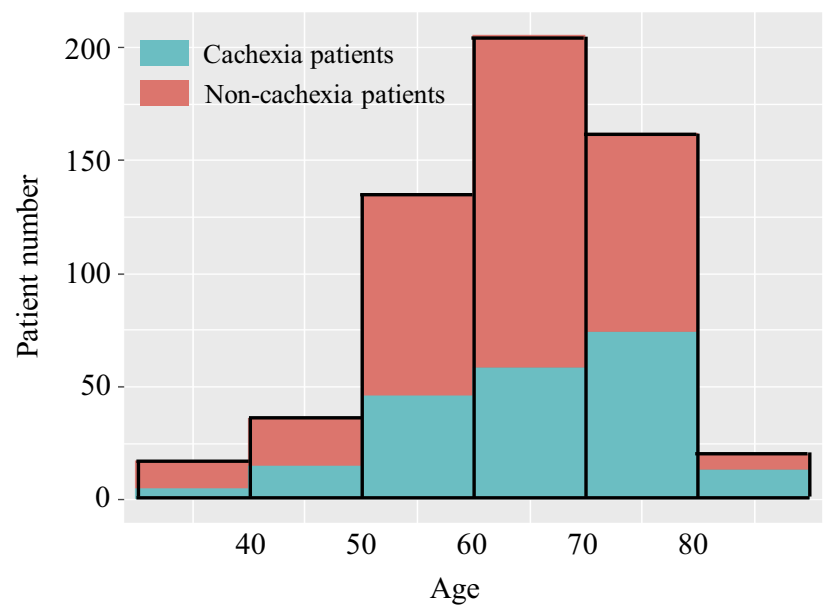

Figure 2 Frequency distribution of patients of different ages stratum with and without cachexia. group I (HR 4.947, 95\% CI 1.181-20.727, $P=0.029)$ than the other three groups (group II: HR 2.232, 95\% CI 1.1034.518, $P=0.026$; group III: HR $1.806,95 \%$ CI 1.052-3.098, $P=0.032$; group IV: HR $1.411,95 \%$ CI $0.915-2.174$, $P=0.119$ ). Further, cachexia only significantly affected postoperative length of stay and hospitalization costs in group I; no such differences were found in the other three groups (Figure 3B).

\section{Discussion}

Cancer cachexia is a multifactorial syndrome, primarily defined as a complex metabolic syndrome associated with underlying illness and characterized by loss of muscle. ${ }^{8}$ Historically, cachexia was thought of as a syndrome of anorexia, fatigue, and weight loss. However, it was recently redefined precisely as lean muscle mass loss associated with chronic illness and/or cancer. ${ }^{9}$ Using the corrected definition, the prevalence of cachexia was 35.8\% in this prospective study. A previous study including patients with several cancer types investigated the association between cachexia and hospitalization costs in the United States, as well as length of stay, and found that the prevalence of cachexia in GC patients was $7.49 \%,{ }^{7}$ much lower than that in our study. It is possible that the previous study did not use the consensus diagnostic criteria for cachexia. Further, ethnic differences may contribute bias to some extent.

The present study prospectively investigated the impact of preoperative cachexia on postoperative OS in GC patients. Our results demonstrated that OS was significantly shorter in patients with preoperative cachexia, and cachexia was found to be an independent risk factor of OS. The relationship between cachexia and disease outcome could probably be explained by the effects of inflammation and malnutrition. Previous studies have suggested a possible association between cachexia and systemic inflammation. ${ }^{10}$ We found that the NLR and PLR (for which a high value is associated with higher systemic inflammation) were both significantly higher in cachexia patients, in accordance with a study reporting that the preoperative NLR and PLR were useful predictors of postoperative survival in patients with stage I-II GC. ${ }^{11}$

Cancer cachexia results not only from reduced nutrient intake or availability, but also from metabolic abnormalities triggered by the tumor, as well as by antineoplastic therapies. These factors stimulate systematic inflammation and cytokine networks ${ }^{12}$ that in turn result in significant loss of body weight, alterations in body composition, and declining 
A
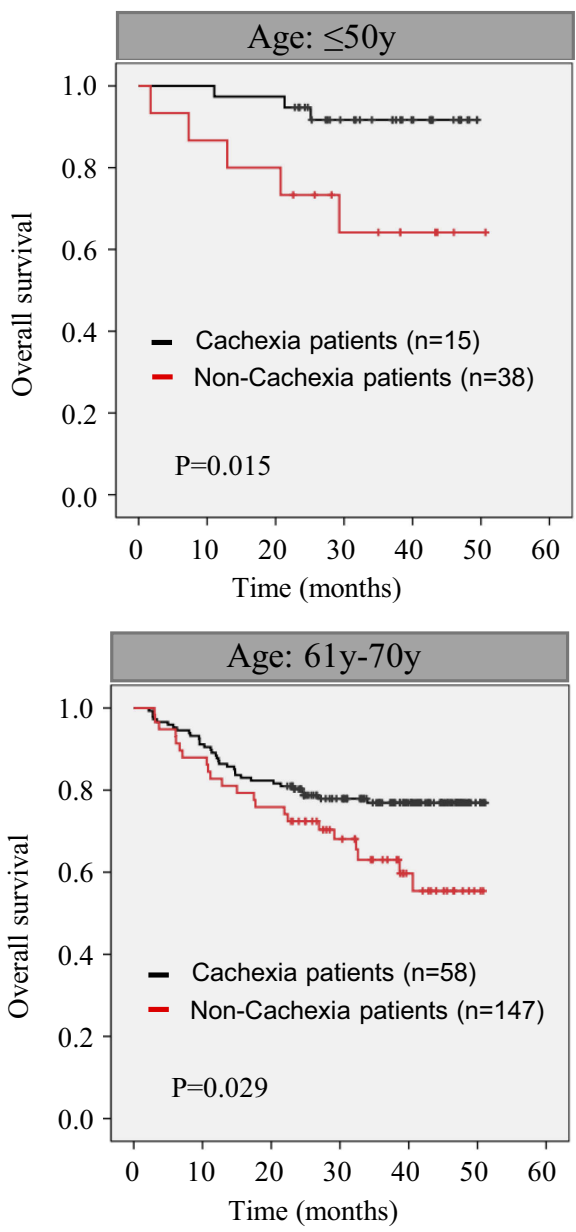

B

帛 Non-Cachexia patients 帛 Cachexia patients

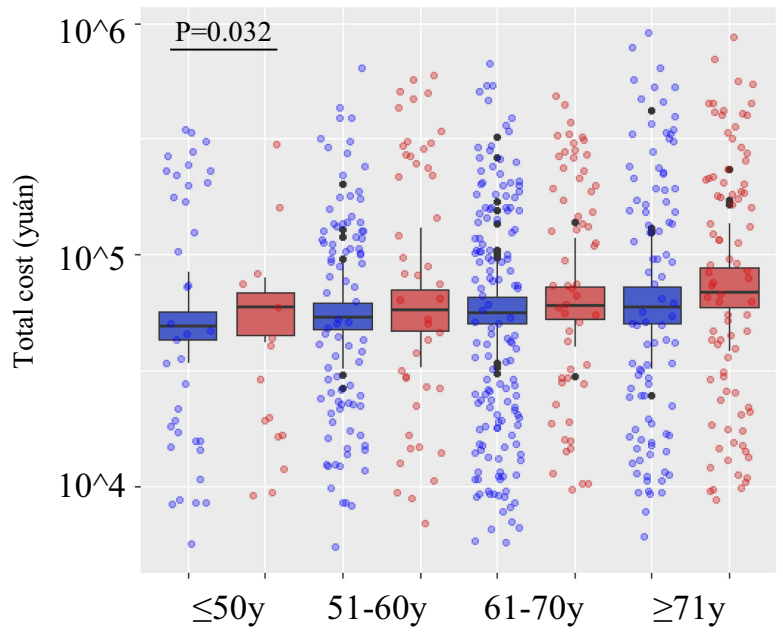

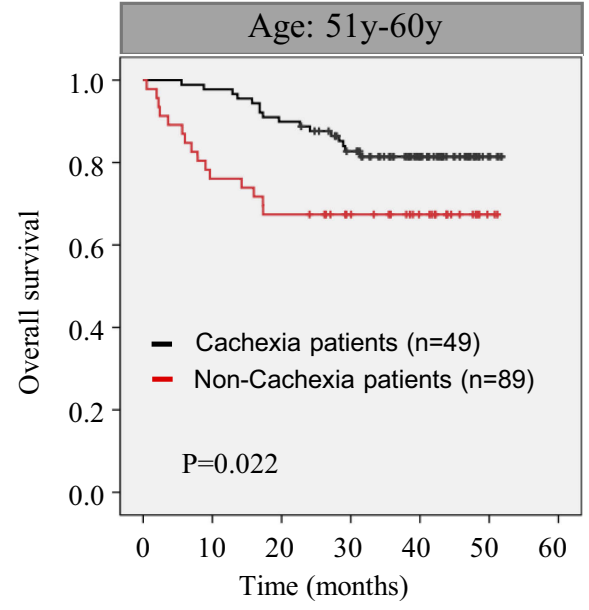
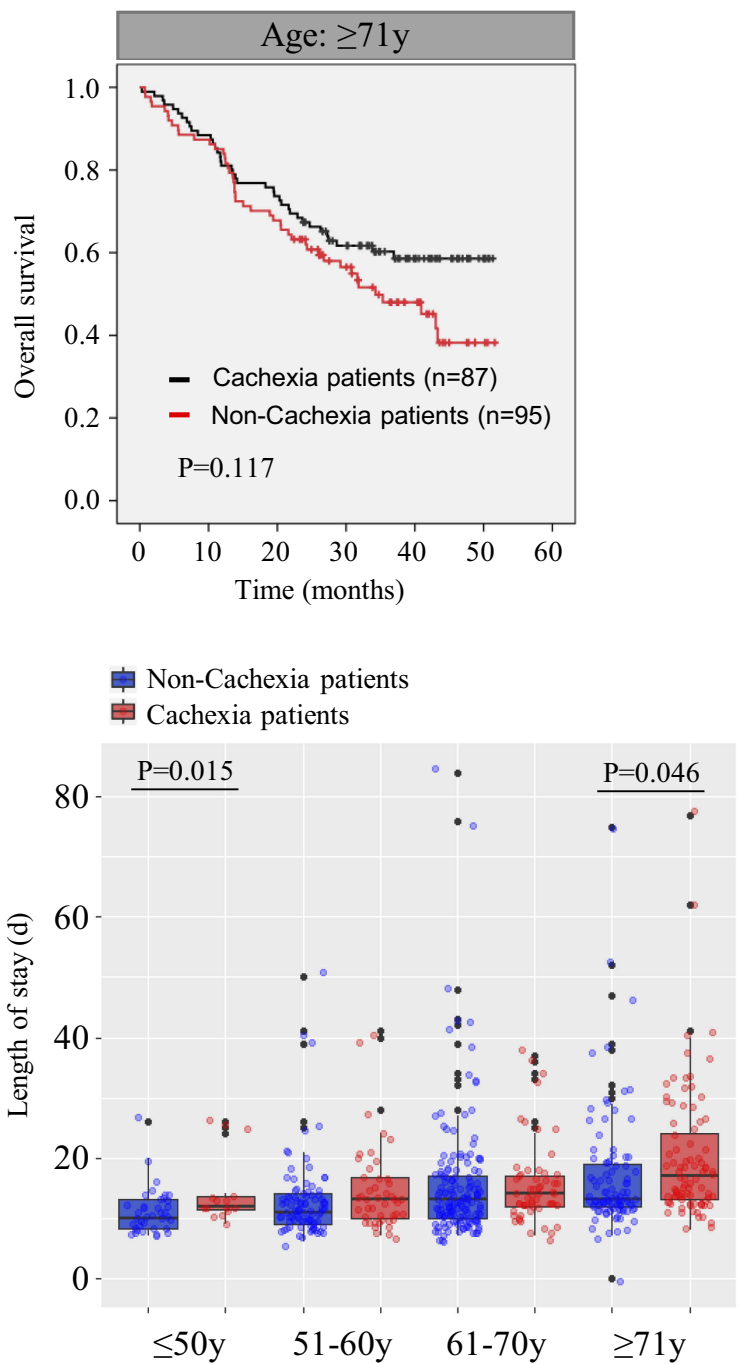

Figure 3 (A) Kaplan-Meier curve for overall survival (OS) in different ages stratum with and without cachexia. (B) Total cost and length of hospital stay in different ages stratum with and without cachexia.

physical function. Our findings showed that cachexia patients were more likely to be thinner, with a lower BMI; they also presented with less skeletal muscle, worse muscle function, and worse basic physical condition (higher ASA stage and $\mathrm{CCI}$ ). Physical condition is a strong prognostic indicator of patient outcomes, ${ }^{13,14}$ as malnutrition and micronutrient 
Table 3 COX regression analysis of the relationship between OS and cachexia in patients with different ages

\begin{tabular}{|l|l|l|}
\hline Group & HR $(95 \% \mathrm{Cl})$ & $P$-value \\
\hline$\leq 50 \mathrm{y}$ & $4.947(1.18 \mathrm{I}-20.727)$ & $0.029 *$ \\
$5 \mathrm{I}-60 \mathrm{y}$ & $2.232(1.103-4.518)$ & $0.026 *$ \\
$61-70 \mathrm{y}$ & $1.806(1.052-3.098)$ & $0.032^{*}$ \\
$\geq 71 \mathrm{y}$ & $1.411(0.915-2.174)$ & 0.119 \\
\hline
\end{tabular}

Notes: Data are presented as median [IQR]. *Statistically significant $(P<0.05)$.

deficiency can lead to the abnormal function of macrophages, neutrophils, and lymphocytes, which can inhibit the immune response, ${ }^{15}$ decrease response to anticancer therapy, ${ }^{16}$ and even increase the risk of tumor recurrence. ${ }^{17}$

Similar to the results of other studies, ${ }^{7,18}$ the incidence of cachexia was higher in the elderly group. Considering that cachexia was significantly related to age and both cachexia and age were independent risk factors for OS, age and cachexia may interact with each other. Therefore, we subdivided the patients into four age groups and examined whether the correlation between cachexia and outcome was age-dependent. Interestingly, cachexia was a more effective risk factor for survival in the youngest group (younger than 50 years old; $\mathrm{HR}=4.947, P=0.029$ ), and it was not significant in the oldest group (older than 70 years old; HR $=1.411, P=0.119$ ), which was quite different from the findings of previous studies. ${ }^{6,19}$ This may be explained by the fact that the interplay between chronic illness and elements such as malnutrition and immobility results in elderly patients being particularly vulnerable to cachexia. Additionally, considering the same diagnostic criteria and their better fundamental physical condition, younger cachexia patients may have better consumption than elderly cachexia patients. Moreover, cachexia only significantly affected postoperative length of stay and hospitalization costs in younger patients.

This is the first study to focus on cachexia in younger patients, and we found it to be a risk factor for prognosis in that group. We propose that more attention should be paid to improve the cachexia status in younger patients, as it will be more profitable in improving OS and decreasing length and costs of hospitalization. Fortunately, in recent years, data from some intervention studies have shown that cachexia could be potentially managed and reversed by multimodal interventions, including nutrition support, exercise, and drug therapy. ${ }^{20-22}$

This study has several limitations that should not be overlooked. First, all patients enrolled in our study were treated at two hospitals in Wenzhou, and bias in the population selection may be inevitable. Therefore, a large-scale multicenter trial is essential to verify our conclusion. Additionally, all the patients enrolled were Chinese,

The ethnic differences lead to significant differences in the results from the European and US patients. Second, the follow-up period in the present study is less than 5 years, and complete follow-up data need to be further acquired.

\section{Conclusion}

In our prospective study, we found that cachexia was an independent risk factor for OS in GC patients. Additionally, cachexia could predict poor outcome in younger GC patients; therefore, greater attention should be paid to cachexia in younger patients.

\section{Ethics approval and consent to participate}

All participants provided their written informed consent, and the protocol for this study was approved by the ethics committee of The First Affiliated Hospital of Wenzhou Medical University and The Second Affiliated Hospital of Wenzhou Medical University.

\section{Availability of data and materials}

The datasets used during the current study are available from the corresponding author on reasonable request.

\section{Abbreviations}

GC, gastric cancer; ASA, American Society of Anesthesiologists; BMI, body mass index; CI, confidence interval; NLR, neutrophil/lymphocyte ratio; PLR, platelet/ lymphocyte ratio; NRS, nutritional risk screening; TNM, tumor, lymph node, metastasis.

\section{Acknowledgments}

The authors thank all the participants in this study and the members of our research team. This study was funded by the Department of Health of Zhejiang Province, China (grant no. 2016DTA006), and the Wenzhou Municipal Science and Technology Bureau (grant no. Y20150057).

\section{Disclosure}

The authors declare that they have no competing interests in this work. 


\section{References}

1. Fearon K, Strasser F, Anker SD, et al. Definition and classification of cancer cachexia: an international consensus. Lancet Oncol. 2011;12:489-495. doi:10.1016/S1470-2045(10)70218-7

2. Porporato PE. Understanding cachexia as a cancer metabolism syndrome. Oncogenesis. 2016;5:e200. doi:10.1038/oncsis.2016.3

3. Argiles JM, Busquets S, Stemmler B, Lopez-Soriano FJ. Cancer cachexia: understanding the molecular basis. Nat Rev Cancer. 2014;14:754-762. doi:10.1038/nrc3829

4. Mondello P, Lacquaniti A, Mondello S, et al. Emerging markers of cachexia predict survival in cancer patients. BMC Cancer. 2014;14:828. doi:10.1186/1471-2407-14-828

5. Bachmann J, Buchler MW, Friess H, Martignoni ME. Cachexia in patients with chronic pancreatitis and pancreatic cancer: impact on survival and outcome. Nutr Cancer. 2013;65:827-833. doi:10.1080/ 01635581.2013 .804580

6. Fukuta A, Saito T, Murata S, et al. Impact of preoperative cachexia on postoperative length of stay in elderly patients with gastrointestinal cancer. Nutrition. 2018;58:65-68. doi:10.1016/j.nut.2018.06.022

7. Arthur ST, Van Doren BA, Roy D, et al. Cachexia among US cancer patients. J Med Econ. 2016;19:874-880. doi:10.1080/ 13696998.2016.1181640

8. Evans WJ, Morley JE, Argiles J, et al. Cachexia: a new definition. Clin Nutr. 2008;27:793-799. doi:10.1016/j.clnu.2008.06.013

9. Prado CM, Sawyer MB, Ghosh S, et al. Central tenet of cancer cachexia therapy: do patients with advanced cancer have exploitable anabolic potential? Am J Clin Nutr. 2013;98:1012-1019. doi:10.3945/ ajcn.113.060228

10. MF N. Cachexia - an intrinsic factor in wound healing. Int Wound $J$. 2010;7:107-113. doi:10.1111/j.1742-481X.2010.00663.x

11. Sun X, Liu X, Liu J, et al. Preoperative neutrophil-to-lymphocyte ratio plus platelet-to-lymphocyte ratio in predicting survival for patients with stage I-II gastric cancer. Chin J Cancer. 2016;35:57. doi:10.1186/s40880-016-0122-2

12. Fearon K, Arends J, Baracos V. Understanding the mechanisms and treatment options in cancer cachexia. Nat Rev Clin Oncol. 2013;10:90-99. doi:10.1038/nrclinonc.2012.209
13. Zheng HL, Lu J, Li P, et al. Effects of preoperative malnutrition on short- and long-term outcomes of patients with gastric cancer: can we do better? Ann Surg Oncol. 2017;24:3376-3385. doi:10.1245/s10434017-5998-9

14. Zhang Y, Wang JP, Wang XL, et al. Computed tomography-quantified body composition predicts short-term outcomes after gastrectomy in gastric cancer. Curr Oncol. 2018;25:e411-e422. doi:10.3747/ co. 25.4014

15. Cunningham-Rundles S, McNeeley DF, Moon A Mechanisms of nutrient modulation of the immune response. $J$ Allergy Clin Immunol. 2005;115:1119-1128; quiz 1129. doi:10.1016/j. jaci.2005.04.036

16. Kizer NT, Thaker PH, Gao F, et al. The effects of body mass index on complications and survival outcomes in patients with cervical carcinoma undergoing curative chemoradiation therapy. Cancer. 2011;117:948-956. doi:10.1002/cncr.25544

17. Moon HG, Han W, Noh DY. Underweight and breast cancer recurrence and death: a report from the Korean Breast Cancer Society. $J$ Clin Oncol. 2009;27:5899-5905. doi:10.1200/JCO.2009.22.4436

18. Karmali R, Alrifai T, Fughhi IAM, et al. Impact of cachexia on outcomes in aggressive lymphomas. Ann Hematol. 2017;96:951956. doi:10.1007/s00277-017-2958-1

19. Naito T, Okayama T, Aoyama T, et al. Unfavorable impact of cancer cachexia on activity of daily living and need for inpatient care in elderly patients with advanced non-small-cell lung cancer in Japan: a prospective longitudinal observational study. BMC Cancer. 2017; 17:800. doi:10.1186/s12885-017-3795-2

20. Ali S, Garcia JM. Sarcopenia, cachexia and aging: diagnosis, mechanisms and therapeutic options - a mini-review. Gerontology. 2014;60:294-305. doi:10.1159/000356760

21. Wen HS, Li X, Cao YZ, et al. Clinical studies on the treatment of cancer cachexia with megestrol acetate plus thalidomide. Chemotherapy. 2012;58:461-467. doi:10.1159/000346446

22. Stewart Coats AJ, Ho GF, Prabhash K, et al. Espindolol for the treatment and prevention of cachexia in patients with stage III/IV non-small cell lung cancer or colorectal cancer: a randomized, double-blind, placebo-controlled, international multicentre phase II study (the ACT-ONE trial). J Cachexia Sarcopenia Muscle. 2016;7:355365. doi: $10.1002 / \mathrm{jcsm} .12126$

\section{Publish your work in this journal}

Cancer Management and Research is an international, peer-reviewed open access journal focusing on cancer research and the optimal use of preventative and integrated treatment interventions to achieve improved outcomes, enhanced survival and quality of life for the cancer patient.
The manuscript management system is completely online and includes a very quick and fair peer-review system, which is all easy to use. Visit http://www.dovepress.com/testimonials.php to read real quotes from published authors. 